Bundesgesundheitsbl -

Gesundheitsforsch - Gesundheitsschutz

2002 • 45:332-337 @ Springer-Verlag 2002

Originalien und Übersichtsarbeiten

A. Dippelhofer • C. Meyer • P. Kamtsiuris • G. Rasch • S. Reiter • K.E. Bergmann

Robert Koch-Institut, Berlin

\title{
Erste Ergebnisse zum Impfstatus aus der Pilotphase des Kinder- und Jugend- gesundheitssurveys
}

aller Altersgruppen aussagekräftig ermittelt. Soziodemografische Charakteristika, die mit fehlendem Impfschutz einhergehen, können aufgezeigt werden. Mit Hilfe der serologischen Untersuchungen kann die Immunität auch dann bestimmt werden, wenn der Impfpass nicht vorliegt (s. auch [7]: Bei den Schuleingangsuntersuchungen besitzen $13 \%$ der Kinder keinen Impfausweis. In der Studie von Koch et al. [3] fehlt die Impfdokumentation bei 3,8 \% der ostdeutschen sowie $8,6 \%$ der westdeutschen Teilnehmer). Weiterhin erlaubt die Erhebung einen Einblick in die altersbezogene Entwicklung des Impfschutzes und gibt Aufschluss über Impfversager oder das Abnehmen der Immunität über die Zeit. Diese Informationen sind besonders bei den neueren Impfungen von Bedeutung. Hierzu zählt die erst 1998 eingeführte Impfung mit dem inaktivierten Polioimpfstoff, die seit 1995 generell empfohlene Impfung gegen Hepatitis B und die Impfung mit dem azellulären Pertussisimpfstoff. Serologische Untersuchungen an einem nicht repräsentativen Kollektiv von Restseren, die 1995 gesammelt wurden, haben gezeigt, dass vor allem im Kleinkindalter bedeutende Impflücken bestehen [8].

\section{Probanden und Methoden}

Über einen Zeitraum von einem Jahr (März 2001 bis März 2002) wurden in der Pilotphase des geplanten bundesweit repräsentativen Kinder- und Jugendge- sundheitssurveys 1700 Kinder und Jugendliche im Alter von o bis 17 Jahren untersucht. Die Studie diente vor allem der Erprobung der Methoden und des Studiendesigns. Dies hat bestimmte methodische Einschränkungen erforderlich gemacht, die zu systematischen Verzerrungen geführt haben könnten. Von Bedeutung ist in diesem Zusammenhang insbesondere die Testung von unterschiedlichen Feldzugängen (Stichproben aus Schuluntersuchungen und Stichproben aus dem Einwohnermelderegister), was zu einer ungleichen Verteilung in den einzelnen Altersklassen geführt hat. Außerdem beschränkte sich die Erhebung auf vier Untersuchungspunkte (Berlin-Steglitz, Berlin-Friedrichshain, Wesendorf in Niedersachsen und Neuruppin in Brandenburg) und kann damit nicht als repräsentativ für die Bundesrepublik Deutschland angesehen werden. Hinzu kommt, dass trotz relativ hoher Responseraten von über $60 \%$ (Einwohnermelderegisterstichprobe) bzw. über $80 \%$ (Schulstichprobe) eine überproportionale Teilnahme von Kindern, deren Eltern an Gesundheitsthemen besonders interessiert sind und damit auch Impfungen häufiger in Anspruch nehmen, nicht ausgeschlossen werden kann.

Angela Dippelhofer Robert Koch-Institut, Nordufer 20,13353 Berlin, E-Mail:dippelhofera@rki.de 
Der Impfstatus der Kinder und Jugendlichen wurde im Rahmen eines computerassistierten ärztlichen Interviews erhoben. In einer Abfragemaske wurde das Vorliegen bzw. Fehlen eines Impfausweises festgehalten und die Anzahl der dort dokumentierten Impfungen gegen Diphtherie, Tetanus, Polio, Pertussis, Hämophilus influenzae, Masern, Mumps, Röteln und Hepatitis B erfasst. Zusätzlich wurden vertiefende Fragen zur Ursache nicht erfolgter Impfungen und zu möglichen Impfreaktionen gestellt. Insgesamt konnten nur 6,9\% der Kinder und Jugendlichen keinen Impfausweis vorlegen. Dies betraf vor allem die über den Schulzugang untersuchten und damit älteren Kinder. In der Altersgruppe der Zwei- bis Sechsjährigen fehlten lediglich 3,5\%, bei den Sieben- bis Elfjährigen sogar nur 1,9\% der Impfausweise.
Im Verlauf des Pretests wurde deutlich, dass das dargelegte Erfassungsverfahren nicht ausreicht, um Aussagen zu Qualität und Zeitgerechtigkeit der Impfungen zu machen. Aus diesem Grund wurde die Erhebungsmaske modifiziert, so dass nun eine Unterscheidung zwischen Grundimmunisierung und Auffrischimpfung möglich ist und die Zeitgerechtigkeit erfasst werden kann. Die Ergebnisse, die aus dieser differenzierteren Abfragemaske resultieren, sind jedoch noch nicht Bestandteil der vorliegenden ersten Auswertung.

In dem vorliegenden Bericht wurden die Daten, die an 857 Kindern ab dem vollendeten zweiten Lebensjahr erhoben wurden, verwendet. Unterteilt in drei Altersklassen wurden diese hinsichtlich der Vollständigkeit der Impfungen, dem Bildungsstand der Mutter und stratifiziert nach neuen und alten

Tabelle 1

Definition der Vollständigkeit einer Impfung. Eine Impfung gilt dann als vollständig, wenn die Anzahl der angegebenen Impfungen durchgeführt wurde.

\begin{tabular}{lllllll} 
Altersklasse & D/T & Polio & Pertussis & HIB & MMR & Hepatitis B \\
\hline 2 - 6 Jahre & $\geq 3$ & $\geq 3$ & $\geq 4$ & $\geq 3$ & $\geq 1$ & $\geq 3$ \\
7 - 11 Jahre & $\geq 4$ & $\geq 3$ & $\geq 4$ & $\geq 3$ & $\geq 2$ & $\geq 3$ \\
12 - 17 Jahre & $\geq 5$ & $\geq 4$ & $\geq 4$ & & $\geq 2$ & $\geq 3$ \\
\hline
\end{tabular}

Impfungen gegen:D/T: Diphtherie/Tetanus, Polio: Poliomyelitis, Pertussis: Keuchhusten, HiB: Hämophilus influenza Typ B, MMR: Masern-Mumps-Röteln.

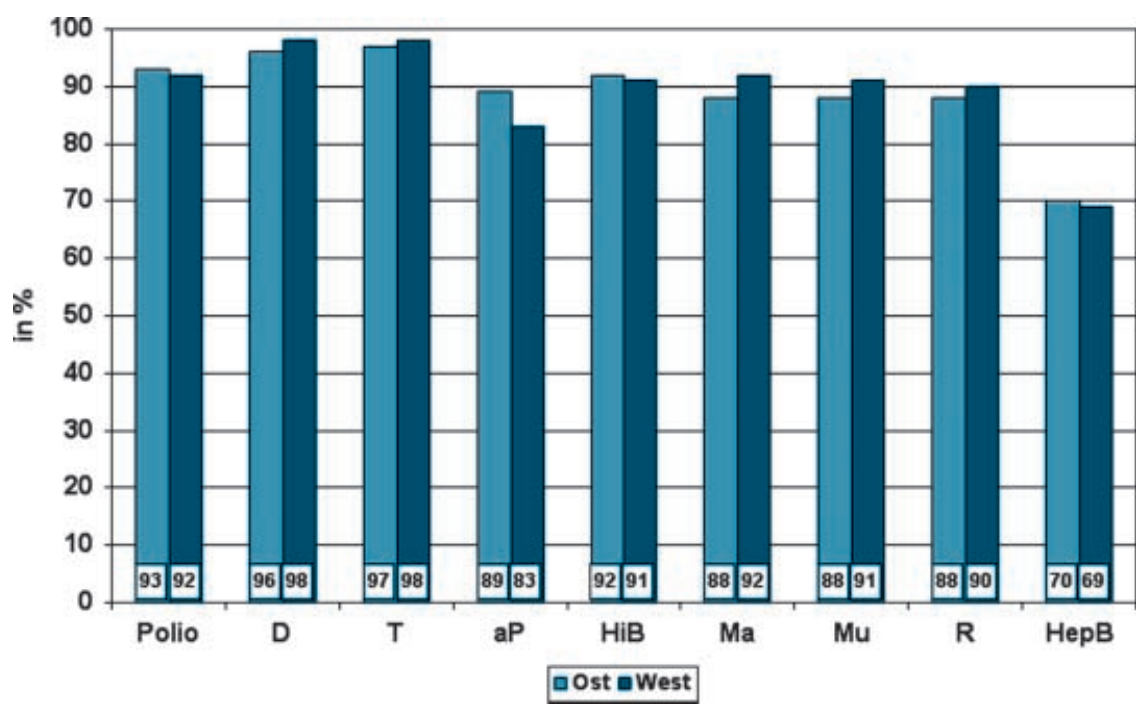

Abb. 1 A Durchimpfungsraten bei Zwei- bis Sechsjährigen. Impfungen gegen: Polio: Poliomyelitis, D: Diphtherie, T: Tetanus, P: Pertussis (aP: Pertussis-Antigen), HiB: Hämophilus influenza Typ B, Ma: Masern, Mu: Mumps, R: Röteln, Hep B: Hepatitis B
Bundesländern ausgewertet. Nach Abschluss der Feldphase werden die Daten sowohl quantitativ (insgesamt werden 1700 Kinder untersucht) als auch qualitativ (differenziertere Erhebungsmaske) erweitert. Bundesweite Repräsentativität wird nach Abschluss der Hauptphase erreicht.

Es wurden drei Altersklassen gebildet: die Altersklassen der Zwei- bis Sechsjährigen, der Sieben- bis Elfjährigen und der Zwölf- bis 17-Jährigen. Die Vollständigkeit einer Impfung wurde nach der Anzahl der durchgeführten Impfungen definiert (Tabelle 1).

Die Zuordnung der Schichtzugehörigkeit erfolgt über den Ausbildungsstand der Mutter. Dabei werden Mütter ohne Schulabschluss oder mit Hauptund Grundschulabschluss der sozial niedrigeren Schicht zugeordnet, Mütter mit Realschul- bzw. POS-Abschluss (Abschluss der Polytechnischen Oberschule) repräsentieren die mittlere Schicht, die obere Schicht wird von Müttern mit Fachhochschulreife bzw. mit Abitur oder EOS-Abschluss (Abschluss der Erweiterten Oberschule) gebildet. Differenzierte Aussagen sind durch die Auswertung mit einem Schichtindex zu erwarten. Die Bildung dieses Index ist derzeit Gegenstand intensiver Forschung und Diskussion.

\section{Ergebnisse}

\section{Durchimpfungsraten nach Altersgruppen und Stratifizierung in neue und alte Bundesländer}

Die Durchimpfungsraten nach Altersgruppen in den neuen und alten Bundesländern sind in Abb. 1-3 dargestellt.

\section{Diphtherie und Tetanus}

Für Diphtherie und Tetanus werden bei den zwei- bis sechsjährigen Kindern Durchimpfungsraten von über $96 \%$ erreicht, bei den Sieben- bis Elfjährigen sind es noch mindestens $94 \%$, wobei keine wesentlichen Unterschiede zwischen den alten und neuen Bundesländern zu verzeichnen sind. Weniger gut sind die Impfraten für die zwölf- bis 17jährigen Kinder und Jugendlichen. Ursache ist die fehlende Umsetzung der empfohlenen Auffrischimpfung. Im Osten werden weiterhin hohe Durchimpfungsraten von $95 \%$ erzielt, während im Westen in dieser Altersklasse nur noch 


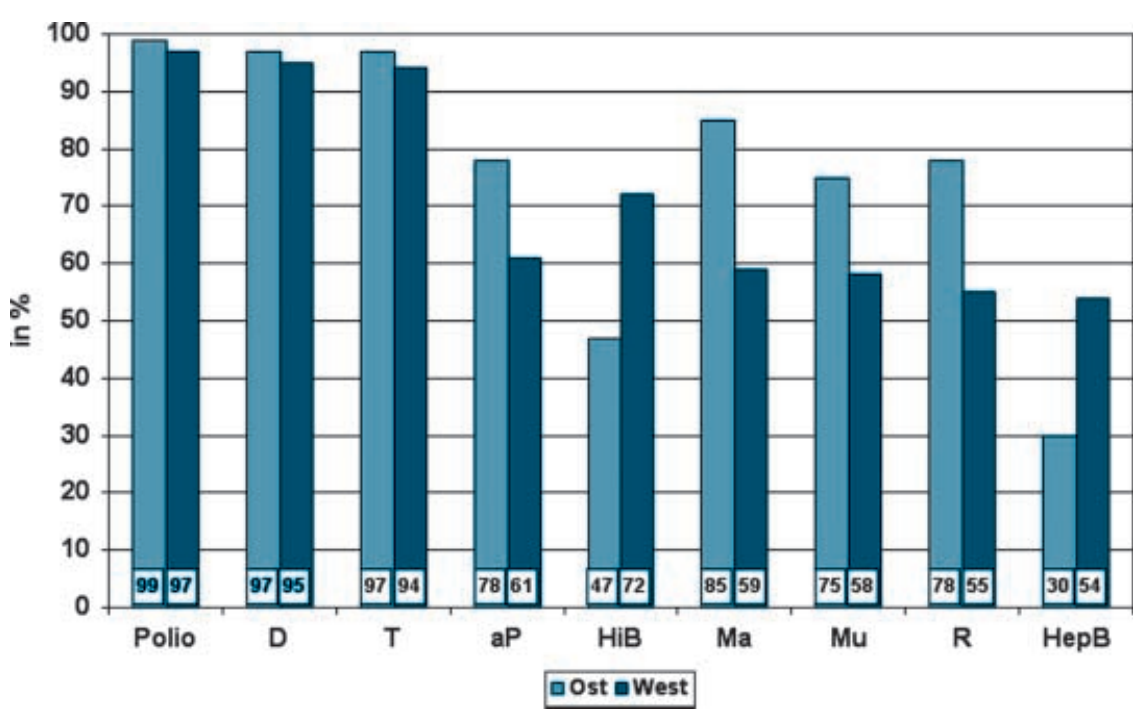

Abb. 2 \ Durchimpfungsraten bei Sieben- bis Elfjährigen. Impfungen gegen: Polio: Poliomyelitis, D: Diphtherie, T: Tetanus, P: Pertussis (aP: Pertussis-Antigen), HiB: Hämophilus influenza Typ B, Ma: Masern, Mu: Mumps, R: Röteln, Hep B: Hepatitis B

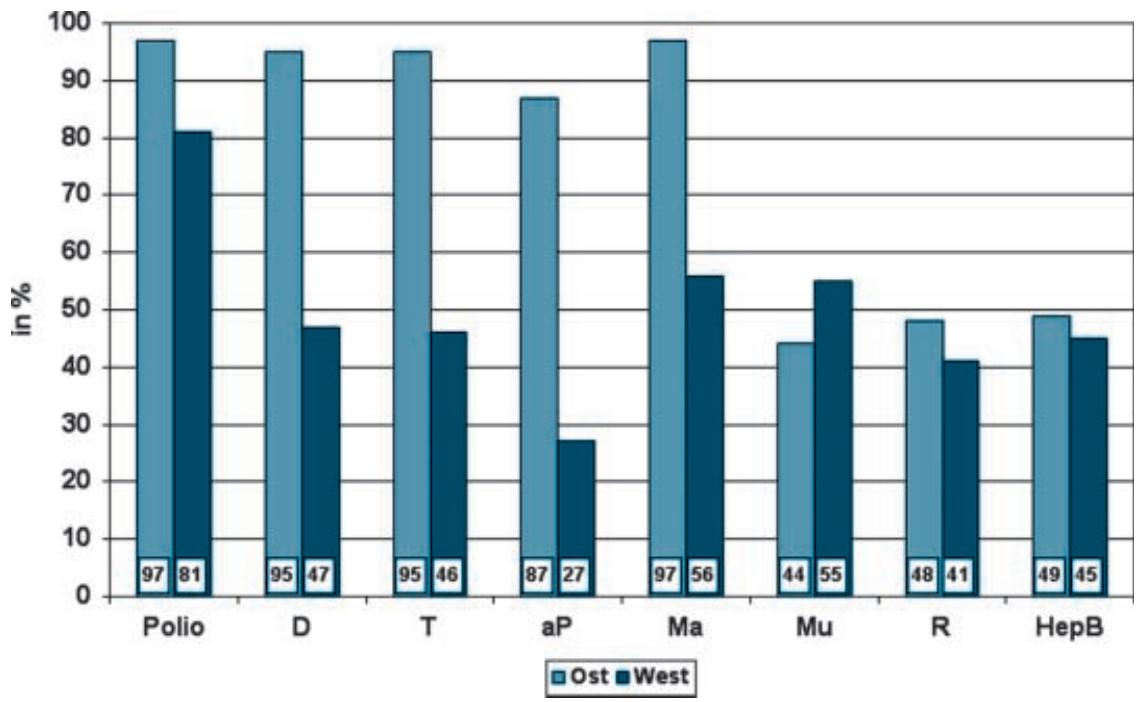

Abb. 3 Durchimpfungsraten bei Zwölf- bis 17-Jährigen. Impfungen gegen: Polio: Poliomyelitis, D: Diphtherie, T: Tetanus, P: Pertussis (aP: Pertussis-Antigen), HiB: Hämophilus influenza Typ B, Ma: Masern, Mu: Mumps, R: Röteln, Hep B: Hepatitis B

$47 \%$ der Kinder und Jugendlichen ausreichend gegen Diphtherie bzw. $46 \%$ gegen Tetanus geimpft sind.

\section{Polio}

Auch für die Impfung gegen Polio sind in den unteren Altersgruppen (zwei bis elf Jahre) hohe Durchimpfungsraten ohne Ost-West-Gefälle zu verzeichnen.
Pertussis

Bei den zwei- bis sechsjährigen Kindern wurden inzwischen mit $89 \%$ im Osten und $83 \%$ im Westen erfreulich hohe, wenn auch noch nicht ausreichende Durchimpfungsraten erreicht. Der bekannte Ost-West-Unterschied stellt sich auch hier dar, ist aber im Vergleich $\mathrm{zu}$ den älteren Altersgruppen geringer geworden. Die Sieben- bis Elfjährigen sind in den neuen Bundesländern zu $78 \%$, in den alten $\mathrm{zu} 61 \%$ gegen Keuchhusten geimpft. Am deutlichsten ist der Unterschied bei den Zwölf- bis 17-Jährigen. Hier sind $87 \%$ der Kinder und Jugendlichen aus dem Osten und nur $27 \%$ aus dem Westen gegen Pertussis geimpft. Ursache dafür ist, dass für das gesamte Bundesgebiet erst seit 1991 eine generelle Impfempfehlung vorliegt.

\section{Hepatitis B}

Die Durchimpfungsraten für Hepatitis B sind in der Altersgruppe der Zwei- bis Sechsjährigen mit ca. $70 \%$ am höchsten (kein Unterschied zwischen alten und neuen Bundesländern), wobei diese Impfrate keinesfalls schon als optimal gelten kann. In den beiden anderen Altersgruppen liegen sie zwischen $30 \%$ und $54 \%$, wobei sich der Ost-West-Unterschied bei den Sieben- bis Elfjährigen dieses Mal umgekehrt darstellt.

\section{Masern, Mumps, Röteln}

Eine steigende Tendenz ist bei den Durchimpfungsraten für die einmalige Masern-Mumps-Röteln-Impfung (MMRImpfung) zu beobachten. Ohne wesentlichen Ost-West-Unterschied liegen diese in der Altergruppe der zwei- bis sechsjährigen Kinder bei mindestens $88 \%$. Für die angestrebte Eliminierung der Masern reicht das jedoch keinesfalls aus. Von den sieben- bis elfjährigen ostdeutschen Kindern erhielten $85 \%$ die zweite Masernimpfung bzw. $75 \%$ die zweite MMR-Impfung. Bei den westdeutschen Kindern waren es 59\% (komplette MMR-Impfung: $55 \%)$. Noch deutlicher ist der Ost-WestUnterschied bei den Zwölf- bis 17-Jährigen mit einer Rate von $97 \%$ für die zweimalige Masernimpfung in den neuen Bundesländern gegenüber $56 \%$ in den alten. Bei Mumps und Röteln sind die Durchimpfungsraten mit $41 \%$ bis $55 \%$ noch geringer. 
Durchimpfungsraten der Zweibis Sechs- und der Sieben- bis Elfjährigen nach Bildungsstand der Mutter

Betrachtet man den Zusammenhang von Durchimpfungsraten und Zugehörigkeit $\mathrm{zu}$ einer sozialen Schicht, zeigen sich Unterschiede in Abhängigkeit vom Alter der Probanden sowie von der die Impfung betreffenden Erkrankung (Abb. 4 und 5). Für die Zwei- bis Sechsjährigen scheinen die Auswirkungen der Schichtzugehörigkeit noch relativ gering $\mathrm{zu}$ sein, wobei in diesem Alter für Unterund Mittelschichten fast grundsätzlich bessere Durchimpfungsraten als für die Oberschicht beobachtet werden. Besonders deutlich ist die geringere Inanspruchnahme von Impfungen durch die Oberschicht für die Masern-, Mumpsund Rötelnimpfung. Sind hier zwischen Unter- und Mittelschichtkindern Spannen von $91 \%$ bis $96 \%$ festzustellen, so

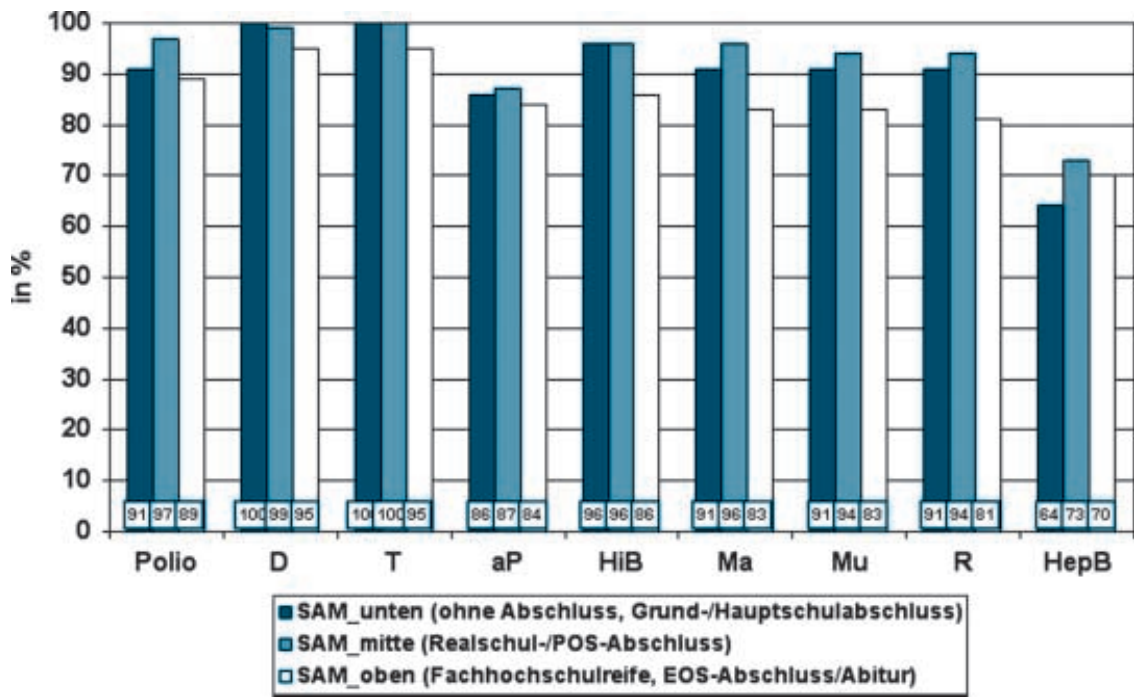

Abb. $4 \Delta$ Durchimpfungsraten bei Zwei- bis Sechsjährigen und Schulabschluss der Mutter. Impfungen gegen: Polio: Poliomyelitis, D: Diphtherie, T: Tetanus, P: Pertussis (aP: Pertussis-Antigen), HiB: Hämophilus influenza Typ B, Ma: Masern, Mu: Mumps, R: Röteln, Hep B: Hepatitis B, SAM: Schulabschluss der Mutter, POS: Polytechnische Oberschule, EOS: Erweiterte Oberschule

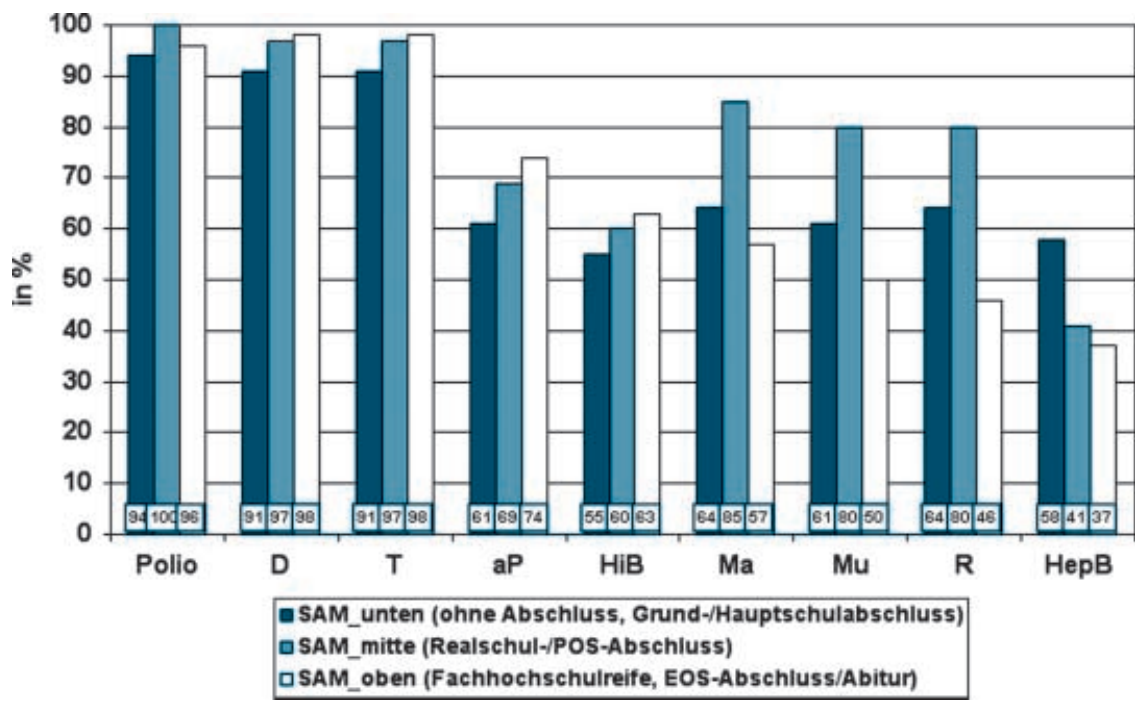

Abb. $5 \Delta$ Durchimpfungsraten bei Sieben- bis Elfjährigen und Schulabschluss der Mutter. Impfungen gegen: Polio: Poliomyelitis, D: Diphtherie, T: Tetanus, P: Pertussis (aP: Pertussis-Antigen), HiB: Hämophilus influenza, Typ B, Ma: Masern, Mu: Mumps, R. Röteln, Hep B: Hepatitis B, SAM: Schulabschluss der Mutter, POS: Polytechnische Oberschule, EOS: Erweiterte Oberschule sind in der Oberschicht nur $81 \%$ bis $83 \%$ der Kinder durch eine Impfung gegen Masern geschützt. Einzig für die Hepatitis-B-Impfung zeigt sich in der Altersgruppe der Zwei- bis Sechsjährigen eine niedrigere Inanspruchnahme durch Kinder aus unteren sozialen Schichten.

Für die Sieben- bis Elfjährigen stellt sich der Zusammenhang zwischen Impfrate und Schichtzugehörigkeit etwas differenzierter dar. Auch hier sind für die klassischen, relativ gut akzeptierten Impfungen wie die gegen Diphtherie, Tetanus und Polio nur geringe Unterschiede bei den Durchimpfungsraten in Abhängigkeit der Schichtzugehörigkeit $\mathrm{zu}$ beobachten. Allerdings kehrt sich in dieser Altersgruppe der Trend von einer geringeren Inanspruchnahme durch die obere Schicht um zu einer geringeren Inanspruchnahme durch die unteren Schichten.

Sehr deutlich wird in dieser Altersklasse die bereits oben angesprochene Ablehnung der Masern-Mumps-RötelnImpfung durch die oberen Schichten. Hier beträgt die Durchimpfungsrate für die Impfung gegen Masern $57 \%$ und für die Impfung gegen Röteln $46 \%$, während diese für die mittlere Schicht bei ca. $80 \%$ und für die untere Schicht immer noch bei über $60 \%$ liegen. Ein gegenläufiger Trend zeigt sich für die Impfung gegen Hepatitis B. Anders als die jüngeren Kinder (siehe oben) sind die älteren Kinder aus den unteren sozialen Schichten häufiger als Mittel- und Oberschichtkinder gegen Hepatitis B geimpft.

\section{Besonders problematische Impfungen (Masern und Pertussis) in Hinblick auf Akzeptanz und Durchimpfung}

\section{Masern}

Abbildung 6 verdeutlicht, dass nur $28 \%$ der Kinder im Westen bzw. 33 \% der Kinder im Osten bis zum vollendeten zweiten Lebensjahr durch eine Impfung gegen Masern geschützt sind. In der Altersgruppe der Zwei- bis Dreijährigen sind in den alten Bundesländern $91 \%$ geschützt, in den neuen aber nur $67 \%$. Für die vorliegende Stichprobe zeigt sich eine zufriedenstellende Durchimpfungsrate für die Drei- bis Sechsjährigen mit durchschnittlichen Raten zwischen $92 \%$ und $94 \%$. Der Abfall der Kurve im Alter von sieben Jahren zeigt die fehlen- 
de Realisierung der zweiten MasernMumps-Röteln-Impfung. In den neuen Bundesländern ist die zweite MMRImpfung grundsätzlich besser akzeptiert und implementiert als in den alten, wobei aber in der Altersgruppe der Sieben- bis Elfjährigen auch für diese Länder eine mit zunehmendem Alter leicht steigende Tendenz zu beobachten ist.

\section{Pertussis}

Für die Pertussisimpfung zeigt sich die bereits für die Masernimpfung beobachtete fehlende Zeitgerechtigkeit noch deutlicher. Hier ist eine 90 \%ige Durchimpfungsrate erst im Alter von drei bis vier Jahren erreicht. Außerdem lassen sich an diesem Beispiel besonders eindrücklich die unterschiedlichen Impf- strategien in der Bundesrepublik und der DDR aufzeigen. Sind in den alten Bundesländern aufgrund der bis 1990 fehlenden Impfempfehlung nur $23 \%$ bis $38 \%$ der Elf- bis 17-Jährigen optimal gegen Pertussis geimpft, so sind es in den neuen Bundesländern immerhin $75 \%$ bis $93 \%$ (Abb. 7 ).

\section{Diskussion}

Vorbehaltlich der oben erörterten methodischen Einschränkungen zeigt sich, dass die Impfbereitschaft deutlich zugenommen hat. Bei den klassischen Impfungen gegen Diphtherie, Tetanus und Poliomyelitis werden insbesondere in den jüngeren Altersgruppen ausreichende Durchimpfungsraten erreicht. In der Altersgruppe der Zwei- bis Sechsjährigen
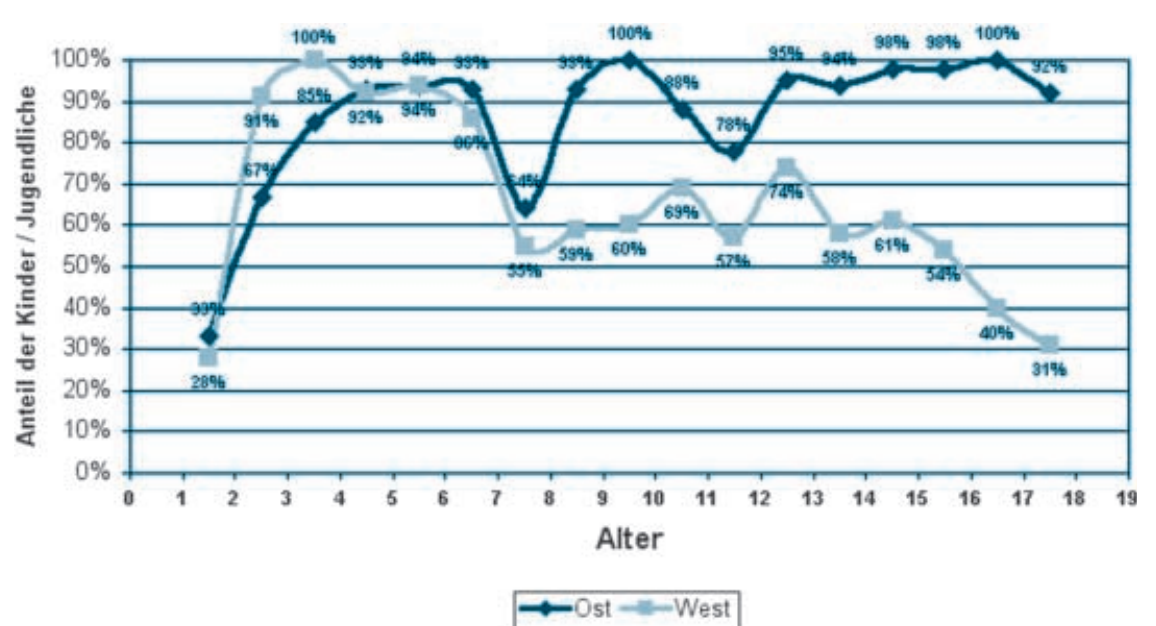

Abb.6 $\Delta$ Alter und Anteil der Kinder/Jugendlichen in den alten (West) und neuen (Ost) Bundesländern, die vollständig gegen Masern geimpft sind

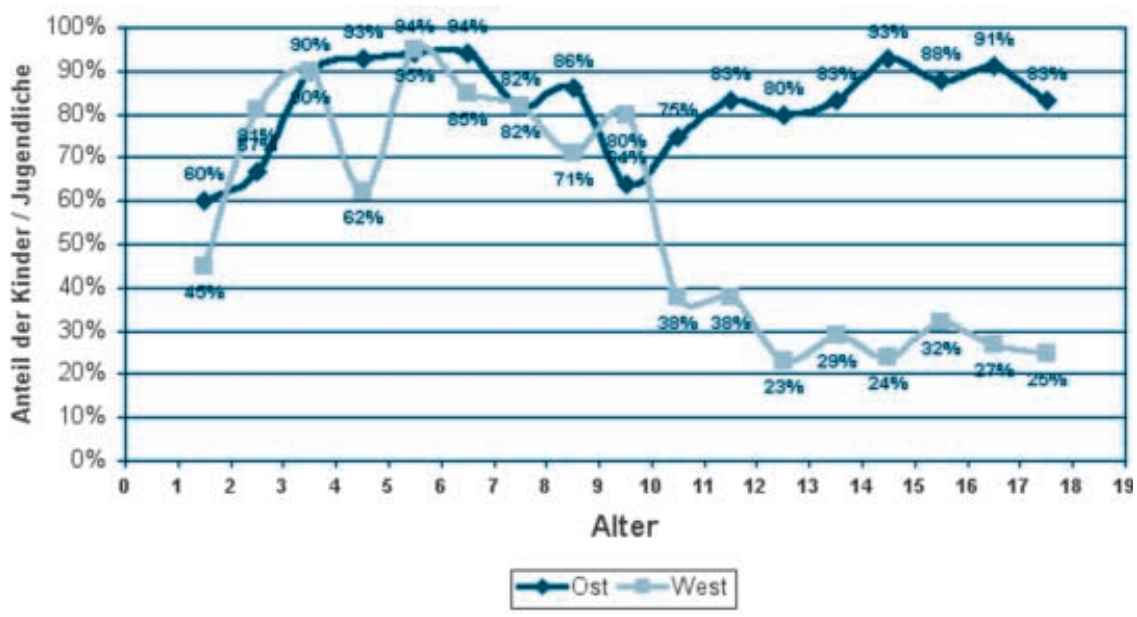

Abb. $7 \Delta$ Alter und Anteil der Kinder/Jugendlichen in den alten (West) und neuen (Ost) Bundesländern, die vollständig gegen Pertussis geimpft sind werden ansteigende Durchimpfungsraten auch für die in der Öffentlichkeit teilweise kritisch bewerteten Impfungen gegen Masern, Mumps und Röteln, Hepatitis B und Pertussis beobachtet. Inwieweit die Einführung von Fünf- und Sechsfachkombinationsimpfungen $\mathrm{zu}$ einem Anstieg der Impfraten beigetragen hat, müssen weitere Analysen zeigen. Sehr erfreulich stellt sich auch die Durchimpfungsrate für die zweite Masern-MumpsRöteln-Impfung im Alter von sieben bis elf Jahren dar. 85\% der Kinder in den neuen Bundesländern und 59\% der Kinder in den alten Bundesländern sind zweimal geimpft. Zum Zeitpunkt der Schuleingangsuntersuchungen der Jahre 1998-2001 [9] waren lediglich 35\% der Kinder in den neuen und sogar nur $16 \%$ in den alten Bundesländern zweimal gegen MMR geimpft. Die Inanspruchnahme der zweiten Masern-Mumps-RötelnImpfung nach Schulbeginn wird wahrscheinlich durch die Aufdeckung der Impflücken aufgrund der Untersuchungen des ÖGDs (öffentlicher Gesundheitsdienst) günstig beeinflusst. Dennoch sind diese Durchimpfungsraten weit von der für eine Maserneradikation erforderlichen Rate von 95\% entfernt.

Die Durchimpfungsraten sind in den neuen Bundesländern überwiegend besser als in den alten Bundesländern. Das gilt sowohl für die klassischen, gut akzeptierten Impfungen gegen Diphtherie, Tetanus und Polio als auch für die von Teilen der Öffentlichkeit kritisch beurteilten Impfungen gegen Masern, Mumps, Röteln und Pertussis. Die Unterschiede werden vor allem bei den Zwölf- bis 17-Jährigen deutlich. Es handelt sich hierbei um noch vor der Wende durch das Gesundheitswesen der DDR versorgte Kinder, aber auch um Kinder, die nach der Wende eine ausreichende Impfauffrischung erhielten. In der Altersgruppe der Zwei- bis Sechsjährigen haben sich die Durchimpfungsraten in Ost- und Westdeutschland angeglichen.

Bemerkenswert sind die niedrigen Polioimpfraten in der Altersklasse der Zwei- bis Sechsjährigen. Es ist zu vermuten, dass diese im Vergleich zu den Sieben- bis Elfjährigen um $2 \%$ bis $3 \%$ geringere Inanspruchnahme darauf zurückzuführen ist, dass die Akzeptanz der Poliomyelitisimpfung in Anbetracht der in der Bundesrepublik Deutschland nicht mehr auftretenden Polioerkrankungen geringer zu werden scheint. 
Die niedrigen Durchimpfungsraten bei Hepatitis B (bei den Sieben- bis Elfjährigen $30 \%$ in den neuen Bundesländern, $54 \%$ in den alten Bundesländern) weisen auf ein Problem hin, das möglicherweise in den bestehenden Versorgungsstrukturen begründet ist. Die Kohorte der Sieben- bis Elfjährigen wurde geboren, bevor die Impfung gegen Hepatitis B im Säuglingsalter allgemein empfohlen wurde. Inhalt der Empfehlung ist auch, dass eine nicht stattgefundene Grundimmunisierung nachzuholen ist. Es gab jedoch in den Anfangsjahren erhebliche Probleme in Hinblick auf die Kostenübernahme durch die Krankenkassen. Zudem bestanden Akzeptanzprobleme vonseiten der Kinderärzte, die durch das Fehlen von Kombinationsimpfstoffen verschärft wurden. Die niedrige Durchimpfungsrate in dieser Altersgruppe spiegelt daher vermutlich das fehlende Nachholen der empfohlenen Impfungen jenseits des Säuglingsalters wider.

Bei der Hypothesengenerierung zur Akzeptanz und Inanspruchnahme von Impfungen in Abhängigkeit von der sozialen Schicht ist zu berücksichtigen, dass mit der Ausbildung der Mutter eine eindimensionale Darstellung der Schichtzugehörigkeit gewählt wurde, die durch weitere systematische Verzerrungen überlagert sein könnte. Vorbehaltlich dieser Einschränkungen wird jedoch deutlich, dass Kinder aus unteren sozialen Schichten insbesondere im Schul- und Jugendlichenalter für fast alle Impfungen eine niedrigere Durchimpfungsrate aufweisen als Mittel- und Oberschichtkinder. Eine Ausnahme bildet jedoch die Durchimpfungsrate gegen Hepatitis B. Sie ist in den unteren sozialen Schichten besser, was eventuell durch den höheren Ausländeranteil dieser Schicht erklärt werden könnte. Ausländische Bürger haben durch den Kontakt zu Regionen mit hoher Prävalenz von Hepatitiserkrankungen eine höhere Expositionsgefahr und daher möglicherweise auch eine größere Akzeptanz für die Impfung. Umgekehrt werden Impfungen, die besonders im Kreuzfeuer der öffentlichen Kritik stehen, von Kindern aus oberen sozialen Schichten seltener in Anspruch genommen. Dies gilt insbesondere für die Impfungen gegen Masern, Mumps und Röteln und in geringerem Maße auch für die Pertussisimpfung.

\section{Literatur}

1. Centers for Disease Control (1998)

Measles, mumps and rubella - vaccine use and strategies: Recommendation of the Advisory Committee on Immunization Practices. MMWR - RR 8:1-67

2. Centers for Disease Control (1999b) Progress toward global poliomyelitis eradication - 1997-1998. MMWR 48(20):416-421

3. Koch J, Kirschner W, Kirschner R, Heydt K (1995) Verbesserung des Kenntnisstandes zur Verbreitung von impfpräventablen Erkrankungen und zum Impfverhalten in der Bundesrepublik Deutschland. Epidemiologische Forschung, Berlin

4. Robert Koch-Institut (2000)

Zur Eliminierung der Masern und der kongenitalen Röteln. Epidemiol Bull 7:53-55

5. WHO Regional Office for Europe (1999) Measles: A strategic framework for elimination of measles in the European region. WHO Regional Office for Europe, Kopenhagen.

6. WHO Regional Office for Europe (1996) Operational targets for EPI diseases. WHO Regional Office for Europe, Kopenhagen

7. Robert Koch-Institut (1999a)

Beginn eines Impfmonitorings in Deutschland: Erhebung von Impfraten zum Zeitpunkt der Einschulung. Epidemiol Bull 23:171-175

8. Gericke E,Tischer A, Santibanez S (2000) Einschätzung der Masernsituation in Deutschland. Bundesgesundheitsbl - Gesundheitsforsch - Gesundheitsschutz 43:11-21

9. Meyer C, Reiter S, Siedler A, Hellenbrand W, Rasch G (2002)

Über die Bedeutung von Schutzimpfungen. Bundesgesundheitsbl - Gesundheitsforsch Gesundheitsschutz; 45:323-337

\section{R. Marantz Henig \\ Der Mönch im Garten.}

\section{Die Geschichte des Gregor Mendel und die Entdeckung der Genetik}

Berlin, Argon Verlag GmbH 2001, $375 \mathrm{~S}$., Abb., geb., EUR 20,40, ISBN 3-87024-528-x

Wenn auch der Titel dieses Buches ein wenig romantisierend klingen mag, so verbirgt sich doch dahinter bei weitem mehr als nur eine „anheimelnde" Erzählung über die Kreuzungsversuche des Gregor Mendel mit Erbsenpflanzen im Klostergarten von Brünn. Über das Leben und Wirken von Gregor Mendel liegen nur wenige Informationen vor, so dass die Autorin es sich erlauben kann, sich „auf das Feld der reinen Spekulation zu begeben, nicht unbedingt nach Herzenslust, schließlich ist dies kein Roman“, und erklärt: „Obwohl ich nicht mit Gewissheit sagen kann, was der Held meines Buches zu einer bestimmten Zeit dachte oder tat, kann ich doch erzählen, wie sich seine Geschichte höchstwahrscheinlich begeben hat, indem ich bestimmte Indizien und geschichtliches Wissen mit einbeziehe." Mit diesem Erzählstil ist es der Autorin in eindrucksvoller Weise gelungen, über den eigentlichen Werdegang des Mönchs Gregor Mendel hinaus seine zeitgeschichtliche Situation und die sich daraus für inn ergebenden Schwierigkeiten einfühlsam darzustellen. Der Autorin macht außerde $m$ deutlich, dass und wie die - aus heutiger Sicht - so einfachen Kreuzungsversuche mit Pisum sativum des einsamen Mönchs im Garten einer der entscheidenden Ausgangspunkte für Genetik und Gentechnik sind. Für diejenigen, die mit der Materie dieser ,Zukunftswissenschaften' nicht so vertraut sind, wird es ein Genuss sein, von leichter Hand geführt einen ersten Überblick über ihre Entstehung zu bekommen; für diejenigen, die sich in diesen,Zukunftswissenschaften' auskennen, mag das Schmunzeln über die menschlichen Unzulänglichkeiten und die persönlichen Anfeindungen im Wissenschaftsbetrieb überwiegen. Ich wünsche dem „Mönch im Garten“ eine große Leserschaft; sie wird mit ihm sehr zufrieden sein.

P. Brandt (Berlin) 